\title{
Withdrawing life-sustaining therapy for patients with severe traumatic brain injury
}

\author{
David H. Livingston MD, Anne C. Mosenthal MD
}

See related research article by Turgeon and colleagues on page 1581 and at www.cmaj.ca/lookup/doi/10.1503/cmaj.101786

Competing interests: None declared.

This article was solicited and has not been peer reviewed.

\section{Correspondence to:} Dr. David H. Livingston, livingst@umdnj.edu

CMAJ 2011. DOI:10.1503 /cmaj.110974
$\mathrm{U}$ ncertainty in prognosis makes the practice of medicine difficult, particularly near the end of life. ${ }^{1}$ This difficulty is compounded by a lack of evidence to guide treatment, leaving physicians with only their best judgment to make clinical decisions. Even in areas of medicine where a strong evidence base exists, significant variations in practice based on geography, ethnicity and insurance status have been reported. It is no surprise that Turgeon and colleagues ${ }^{2}$ found tremendous variations in end-of-life care for patients sustaining severe traumatic brain injury across six trauma centres in Canada.

In their well-crafted analysis, the authors reported several important findings. Overall mortality following severe traumatic brain injury was $31.7 \%$ among the patients involved. However, mortality varied from $10.8 \%$ to $44.2 \%$ across centres. More importantly, over two-thirds of deaths were related to the withdrawal of lifesustaining therapies, with variability ranging from $45.0 \%$ to $86.8 \%$. The statistical modeling employed indicates that more than $20 \%$ of the effect is based on the centre alone, and the variability in mortality was not only associated with, but was affected by, the rates and timing of withdrawal of life-sustaining therapy.

Variability in the practice of withdrawal of life-sustaining therapies between and within medical centres has been well described. It has previously been attributed to racial, ethnic, religious and cultural preferences among patients, as well as the practice patterns of physicians,

\section{KEY POINTS}

- Uncertainty in prognosis and a lack of evidence to guide the treatment of severe traumatic brain injury exist.

- This uncertainty likely drives physicians' communications with families and leads to the large variability in the withdrawal of life-sustaining therapies.

- Communication with families needs to begin early, occur frequently and deliver a consistent message to achieve optimal outcomes and timing of any withdrawal of support.

intensive care units (ICUs), hospitals and regions. There is some evidence that what may appear to be variation in racial preferences are strongly influenced by local hospital "culture" or physician practice. ${ }^{3}$ The palliative care and hospice movement has led to an emphasis on patient preferences and a model of shared decision-making to guide treatment at the end of life. Although we attribute the variability in withdrawal of life-sustaining therapy to differences in patient preferences, the article by Turgeon and colleagues adds to the growing body of literature that physician practice and the culture of medical centres may play an equally strong role.

The large variability seen in end-of-life care between trauma centres must also be understood in the context of the uncertainty that exists in predicting recovery from severe traumatic brain injury. How physicians manage this uncertainty and communicate it to families may account for a large proportion of variability in outcomes. Anecdotal accounts of recoveries of patients who were almost declared brain dead, especially as reported in the lay press, may drive many physicians and families to hold unrealistic expectations. A recent survey found that a high percentage of Americans believe in "miracles" after a severe traumatic injury, regardless of medical prognosis. ${ }^{4}$ Families of patients with critical illnesses take many factors into account in their perception of illness and likelihood of death, with the physicians' prognosis reflecting only a small fraction of their beliefs. ${ }^{5}$

Two reasons for the uncertainty surrounding recovery from traumatic brain injury are the poor discriminatory power of the tools available to measure the extent of brain injury and the lack of outcome data. Age is a significant variable in mortality and functional outcome after traumatic brain injury. In a retrospective review of traumatic brain injury, patients over 65 years of age had a threefold increase in mortality compared with younger cohorts. ${ }^{6}$ In this study, as in the one by Turgeon and colleagues, older age was asso-

All editorial matter in CMAJ represents the opinions of the authors and not necessarily those of the Canadian Medical Association. 
ciated with a higher rate of withdrawal of lifesustaining therapy. In a multicentre prospective study of recovery from isolated traumatic brain injury, older patients had poorer functional status at discharge from hospital and had made less improvement at one year compared with all other patients. ${ }^{7}$ To further explore this issue, we recently conducted a long-term outcome study involving patients with severe injuries who had been admitted to the ICU for more than three days and had survived to discharge. ${ }^{8}$ Despite a poorer overall outcome, one-third of patients with severe traumatic brain injury eventually returned to work. Older age was also a positive variable with respect to patients' overall outlooks toward their disabilities.

Turgeon and colleagues noted variability in the timing of withdrawal of life-sustaining therapy between trauma centres. Although early withdrawal raises the concern of not only hastening death but increasing mortality for patients who might recover with more time, allowing patients to linger when death is inevitable is associated with prolonged suffering for both patients and families. In our prospective study on the impact of an intervention for trauma care focused on communication in the setting of ICU palliative care, we noted earlier withdrawal of life-sustaining therapy and do-not-resuscitate orders. However, no change in either rates of donot-resuscitate orders or mortality was seen among participants. ${ }^{9}$ Age was the greatest predictor of withdrawal of life-sustaining therapy, not the presence of traumatic brain injury.

In the study by Turgeon and colleagues, one half of all deaths occurred within the first three days of care, consistent with the trajectory to brain death in severe traumatic brain injury. However, two-thirds of these deaths were related to the withdrawal of life-sustaining therapy, with a variability of $30.4 \%$ to $92.9 \%$ across centres. This early withdrawal of support may have an impact on organ recovery and donation among patients going on to brain death or organ donation after cardiac death. No data on organ donation was available in this study.

The reasons for withdrawal of life-sustaining therapies are important. For two-thirds of patients, injuries were deemed incompatible with survival; for one-third, withdrawal was related to the expectation of poor neurologic recovery that was incompatible with the patient's wishes. Again, a significant centre effect was noted. It is not clear if some centres withdrew support from patients who were expected to succumb before declaration of brain death, while others allowed patients to proceed to brain death. The trauma centres with lower mortality and a lower rate of withdrawal of life-sustaining therapy, may have discharged patients alive only to shift that mortality to a nursing home.

As the population in western and industrialized countries continues to age and older patients lead healthier and more active lives, the increasing incidence of traumatic brain injury is of great importance. Questions concerning the likelihood and extent of good functional outcomes and costs need to be answered. Turgeon and colleagues are to be commended for their work in this arena. Like most good studies, theirs opens the door to more questions than it could possibly answer.

\section{References}

1. Christakis NA, Lamont EB Extent and determinants of error in doctors' prognoses for terminally ill patients: prospective cohort study. BMJ 2000;320:469-72.

2. Turgeon AF, Lauzier F, Simard J-F, et al. Mortality associated with withdrawal of life-sustaining therapy in patients with severe traumatic brain injury: a Canadian multicentre cohort study. CMAJ 2011 Aug. 29 [Epub ahead of print]

3. Barnato AE, Anthony DL, Skinner J, et al. Racial and ethnic differences in preferences for end-of-life treatment. J Gen Intern Med 2009;24:695-701.

4. Jacobs LM, Burns K, Bennett Jacobs B. Trauma death: views of the public and trauma professionals on death and dying from injuries. Arch Surg 2008;143:730-5.

5. Ford D, Zapka J, Gebregziabher M, et al. Factors associated with illness perception among critically ill patients and surrogates. Chest 2010;138:59-67.

6. Mosenthal AC, Lavery RF, Addis M, et al. Isolated traumatic brain injury: age is an independent predictor of mortality and early outcome. J Trauma 2002;52:907-11.

7. Livingston DH, Lavery RF, Mosenthal AC, et al. Recovery at one year following isolated traumatic brain injury: a Western Trauma Association prospective multicenter trial. J Trauma 2005;59:1298-304.

8. Livingston DH, Tripp T, Biggs C, et al. A fate worse than death? Long-term outcome of trauma patients admitted to the surgical intensive care unit. J Trauma 2009;67:341-8.

9. Mosenthal AC, Murphy PA, Barker LK, et al. Changing the culture around end-of-life care in the trauma intensive care unit. $J$ Trauma 2008;64:1587-93.

Affiliations: David H. Livingston and Anne C. Mosenthal are with the Department of Surgery, New Jersey Medical School, Newark, NJ

Contributors: Both authors contributed to the concept, design, writing and editing of the manuscript. 